The full version of this article may be viewed online at ascopubs.org/journal/jop

\section{Risk Factors and Patterns of Potentially Avoidable Readmission in Patients With Cancer}

Jacques D. Donzé, MD, MSc, Stuart Lipsitz, ScD, MSc, and Jeffrey L. Schnipper, MD, MPH

QUESTION ASKED: What are the risk factors associated with 30-day potentially avoidable readmissions in patients with cancer?

Switzerland; Brigham and Women's Hospital; and Harvard Medical School, Boston, MA

Corresponding author: Jacques D. Donzé, MD, MSc, Department of Medicine, Bern University Hospital, Freiburgstrasse 4, Inselspital, 3001 Bern, Switzerland; e-mail: donze.author@gmail.com.

Disclosures provided by the authors are available with this article at ascopubs.org/journal/jop.
SUMMARY ANSWER: Almost 40\% of patients with cancer had a 30-day readmission, and almost one third of these were deemed potentially avoidable. Risk factors included the number of medications, liver disease, low sodium, and low hemoglobin level at discharge.

WHAT WE DID: In a retrospective cohort of 2,916 patients discharged from the oncology division of an academic tertiary medical center, we identified predictors of potentially avoidable 30-day readmissions by using the SQLape algorithm and multivariable logistic regression.

WHAT WE FOUND: Among the cohort, 37.3\% were readmitted within 30 days, and of these, $31.4 \%$ of all readmissions, $11.7 \%$ of the cohort, were considered potentially avoidable. Several independent risk factors for potentially avoidable readmission were identified. Causes of readmission were similar to the causes of the index readmission: neoplasm, infection, nutritional and metabolic disorders, GI disorders, and renal failure.

BIAS, CONFOUNDING FACTOR(S), REAL-LIFE IMPLICATIONS: Although we were not able to account for the stage of cancer, and we cannot prove preventability of readmissions in these patients, the use of these easily available risk factors can help physicians to identify patients who may benefit from intensive transitional care interventions. 


\title{
Risk Factors and Patterns of Potentially Avoidable Readmission in Patients With Cancer
}

\author{
Jacques D. Donzé, MD, MSC, Stuart Lipsitz, ScD, MSc, and Jeffrey L. Schnipper, MD, MPH
}

Bern University Hospital, Bern,

Switzerland; Brigham and Women's Hospital; and Harvard Medical School, Boston, MA

\section{ASSOCIATED CONTENT}

Appendix DOI: 10.1200/JOP.2016. 011445

DOI: 10.1200/JOP.2016.011445; published online ahead of print at ascopubs.org/journal/jop on October $27,2016$.

\section{Abstract}

\section{Purpose}

Patients with cancer are particularly at risk for readmission within 30-days after discharge. To identify the patients who might benefit from more-intensive discharge interventions, we identified the risk factors associated with 30 -day potentially avoidable readmissions.

\section{Methods and Materials}

We included all consecutive discharges from the oncology division of an academic tertiary medical center in Boston, Massachusetts, between July 1, 2009, and June 30, 2010.

Potentially avoidable 30-day readmissions to the index hospital and two other hospitals within its network were identified. We performed a multivariable logistic regression in which the final model included variables found in bivariable testing to be significantly associated with the outcome.

\section{Results}

Among the 2,916 patients discharged during the study period, 1,086 (37.3\%) were readmitted within 30 days. Of these, 341 (31.4\% of all readmissions, $11.7 \%$ of all discharges) were identified as potentially avoidable. In the multivariable analysis, the following patient factors were associated with a significantly higher risk of a potentially avoidable readmission: total number of medications at discharge, liver disease, last sodium level, and last hemoglobin level before discharge. In addition, potentially avoidable readmissions occurred significantly earlier than unavoidable readmissions (median, 10 v 13 days; $P<.001)$.

\section{Conclusion}

Almost $40 \%$ of patients with cancer had a 30 -day readmission, and almost one third of these were deemed potentially avoidable, and several risk factors for this were identified. Interventions at discharge may be prioritized to patients with these risk factors.

\section{INTRODUCTION}

Avoidable hospital readmission is a widely recognized problem within the modern health care system because of its direct effects on patient outcomes and costs of care and because it represents a natural target for quality improvement efforts. Several studies have identified specific factors associated with hospital readmissions, but emerging data have demonstrated that the nature of the problem is both multifaceted and complex. Underlying diagnoses as well as comorbidities can influence the likelihood of a patient's hospital readmission in ways that remain incompletely understood. ${ }^{1,2}$ Geriatric patients are at increased risk for hospital readmission after an index hospitalization, ${ }^{3}$ but a recent study identified 
patients with cancer as another group at particular risk for readmission within 30 days of hospital discharge. ${ }^{1}$

Evaluation of hospital readmissions within the oncology patient population presents a particular challenge for several reasons. First, many oncology hospitalizations are planned for the administration of elective chemotherapy and do not represent a failure of the system to prevent avoidable readmissions. Second, patient comorbidities are key factors that contribute to avoidable hospital readmissions. ${ }^{1}$ Oncology patients may have multiple and complex comorbidities as a result of not only the etiology of their malignancy but also the expected, and thus incompletely preventable, complications of treatment. Finally, many oncology patients will face end-oflife decisions, another independently identified variable that can contribute to potentially avoidable readmissions. ${ }^{4}$

Overall, oncology patients represent a large and growing complex and medically vulnerable population. Identification of specific risk factors associated with potentially avoidable readmissions in this population may allow for more targeted interventions to prevent readmissions and thereby improve disease outcomes, quality of life, and cost-effective delivery of care. We used a retrospective cohort of consecutive adult patient discharges from an academic tertiary hospital with a large inpatient oncology census to identify specific risk factors associated with 30-day potentially avoidable readmissions.

\section{METHODS AND MATERIALS}

\section{Study Design and Population}

We included all consecutive adult patients discharged from the oncology service of the Brigham and Women's Hospital (BWH)/Dana-Farber Cancer Institute (DFCI) between July 1 , 2009, and June 30, 2010. BWH is a 750-bed academic medical center in Boston, Massachusetts. DFCI is a National Cancer Institute-designated Comprehensive Cancer Center. Since 1997, all DFCI inpatients are hospitalized within BWH. Hospital stays of $\leq 24 \mathrm{~h}$ were not included because they are mainly observational stays. We excluded patients who died before discharge of the index hospitalization, were transferred to another acute health care facility, or left against medical advice. We excluded patients who died during the index hospitalization because they were no longer at risk for readmission, and we excluded patients who were transferred to another health care facility because the risk of readmission must be measured at the time of discharge from the second facility and based on that facility's data. The study protocol was approved by the institutional review board of BWH/ Partners Healthcare.

\section{Study Outcome}

The study outcome was any 30-day potentially avoidable readmission to any service of three hospitals within the Partners Healthcare network, which were BWH, Massachusetts General Hospital (a 1,000-bed tertiary care hospital), and Faulkner Hospital (a 150-bed community hospital closely affiliated with BWH). More than $80 \%$ of all readmissions after an index medical admission to BWH are captured within this network. ${ }^{5,6}$ We identified readmissions deemed potentially avoidable with a validated algorithm (SQLape, Corseaux, Switzerland) that uses administrative data, mainly diagnostic and procedure codes of both index admission and readmission. ${ }^{7,8}$ The algorithm excludes unavoidable foreseen readmissions, such as those for chemotherapy or radiotherapy, transplantation, labor and delivery, and other specific surgical procedures. Follow-up and rehabilitation treatments also are considered unavoidable. Readmissions for a disease that occurred in a new organ system (eg, circulatory, respiratory, digestive, hepatic, nervous, blood) not affected during the index hospitalization were also considered unavoidable. Readmissions related to specific difficult-to-cure diseases (eg, multiple sclerosis, idiopathic thrombocytopenia) were considered unavoidable. Conversely, complications of treatment, such as deep vein thrombosis or catheter-associated urinary tract infection, were considered potentially avoidable, ${ }^{8}$ as were other readmissions that involve an organ system affected during the index hospitalization. The sensitivity and the specificity of the screening algorithm reached $96 \%$ compared with medical chart review (with use of the same criteria) in a random sample of admission-readmission pairs. ${ }^{9}$ We chose the SQLape algorithm because it was the only available tool to our knowledge that differentiates unavoidable from potentially avoidable readmissions.

\section{Predictor Variables}

We collected data on several types of variables from easily obtainable sources (Table 1), including demographic information, previous health care use, primary care provider information, and index admission characteristics from administrative data sources; procedures and chronic medical conditions from billing data; and last known laboratory values before discharge from the Partners Healthcare clinical data 
Table 1. Baseline Characteristics and Bivariable Analysis

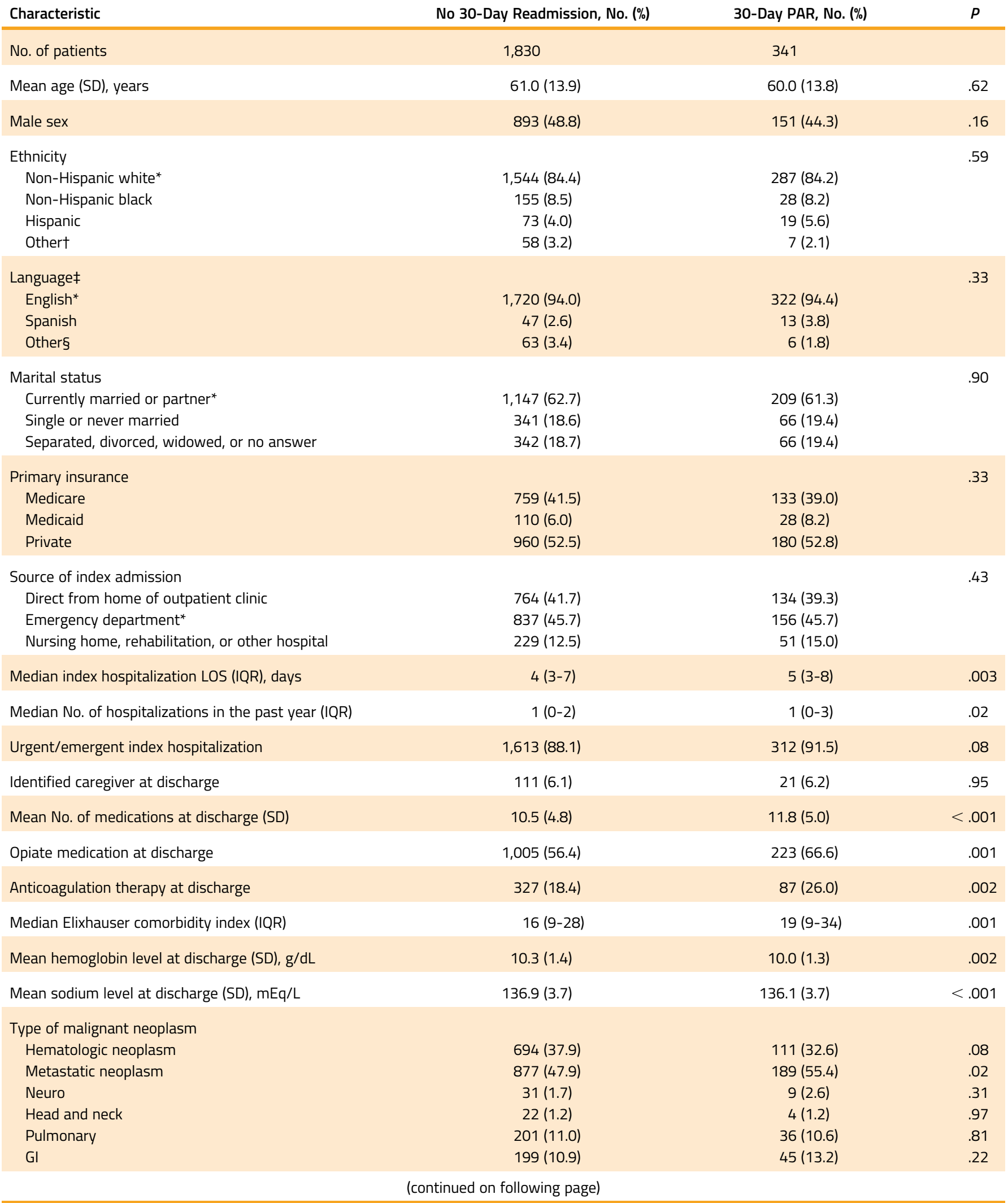


Table 1. Baseline Characteristics and Bivariable Analysis (continued)

\begin{tabular}{lcc} 
Characteristic & No 30-Day Readmission, No. (\%) & 30-Day PAR, No. (\%) \\
\hline Renal & $30(1.6)$ & $7(2.1)$ \\
Gynecologic & $48(2.6)$ & $17(5.0)$ \\
Pancreatic & $52(2.8)$ & $14(4.1)$ \\
Selected comorbidities & & .61 \\
Diabetes mellitus & $280(15.3)$ & .07 \\
Congestive heart failure & $101(5.5)$ & .72 \\
Ischemic heart disease & $182(9.9)$ & $29(8.5)$ \\
Atrial fibrillation & $125(5.8)$ & $39(11.4)$ \\
Chronic obstructive pulmonary disease & $124(6.8)$ & .05 \\
Chronic kidney disease & $106(5.8)$ & .46 \\
Liver disease & $54(3.0)$ & .39 \\
Most frequent reasons of the index admission & & $.68(7.3)$ \\
Neoplasm & & $20(5.9)$ \\
Infectious disease & $435(23.8)$ & $16(4.7)$ \\
Gl disorder & $203(11.1)$ & .96 \\
Nutritional and metabolic disorder & $93(5.1)$ & .07 \\
Liver disorder & $81(4.4)$ & .37 \\
Renal failure & $39(2.1)$ & $66(19.4)$ \\
Venous thromboembolism & $35(1.9)$ & $29(8.5)$ \\
\hline
\end{tabular}

Abbreviations: IRQ, interquartile range; LOS, length of stay; PAR, potentially avoidable readmission; SD, standard deviation.

${ }^{*}$ Reference group for the multivariable logistic regression.

tIncludes Asian, Pacific Islander, Native American, and Alaskan Native.

$\ddagger$ Preferred language based on hospital administrative data.

§lncludes Chinese and French.

repository. Variables were chosen a priori and according to the medical literature. ${ }^{10-12}$

Comorbidities were retrieved from the index hospitalization by using the following International Classification of Diseases, Ninth Revision, Clinical Modification, codes: diabetes mellitus (249.00 to 250.99), congestive heart failure (428.x, 425.4 to $425.9,402.01,402.11$, and 398.91), ischemic cardiac disease (410.00 to 414.99), atrial flutter/fibrillation (427.30 to 427.32), chronic obstructive pulmonary disease (491.00 to 492.99, 493.2, and 496), chronic kidney disease (585.00 to 586.99), and liver disease (571.00 to 573.99).

\section{Primary Diagnosis of Readmission}

The primary diagnosis of each readmission was identified by using the following Medicare Severity Diagnosis-Related Groups codes: congestive heart failure (291 to 293, 189), chronic obstructive pulmonary disease (190 to 192), cardiac ischemic disease ( 231 to 236,246 to 251,280 to 285 ), arrhythmia (308 to 310 ), cerebrovascular diseases (061 to 072), adverse drug events (917 to 923), renal failure (682 to 685), nutritional and metabolic disorders (640 to 641), venous thromboembolism ( 175 to 176,294 to 295 ), liver disorders ( 405 to 434,438 to 446 ), GI disorders (391 to 392,377 to 384 ), infectious diseases ( 075 to 076,094 to 099,121 to 122,177 to 179,193 to 195,371 to 373,485 to 489,548 to 550,689 to 690 , 757 to 759,853 to 863,865 to 872 ), and neoplasm (054 to 055 , 146 to 148,180 to 182,374 to 376,435 to 437,582 to 583,597 to 599,656 to 658,686 to 688,715 to 724,736 to 741,754 to 756 , 846 to 849 ).

\section{Statistical Analysis}

Patient baseline characteristics are presented as proportions, means with standard deviations, and medians with interquartile ranges (IQRs) as appropriate. The unit of analysis was any patient's index discharge from the oncology service.

The presence of any difference in baseline characteristics between the groups with a 30-day potentially avoidable readmission and those not readmitted at all was first tested by bivariable logistic regression. We then performed a multivariable logistic regression in which the final model included variables found to be significantly associated with the outcome in bivariable testing at the $P=.05$ level. The time to 
readmission within the 30-day frame is presented as median with IQR.

All tests were conducted as two sided at a .05 level of significance. Analyses were performed with SAS 9.3 statistical software (SAS Institute, Cary, NC).

\section{RESULTS}

Among the 3,505 patients discharged from the oncology division, 399 were excluded because they were transferred to another acute health care facility, 185 because they died, and five because they left against medical advice. Of the remaining 2,916 patient discharges, $1,086(37.3 \%)$ were followed by a readmission within 30 days. Of these, 341 (31.4\% of all readmissions, $11.7 \%$ of all discharges) were identified as potentially avoidable (Appendix Fig A1, online only). Table 1 compares the baseline characteristics between the patients who did not have a 30-day readmission and those who had a 30-day potentially avoidable readmission.

From the bivariable analysis, the following factors were associated with a 30-day potentially avoidable readmission at the .10 significance level: length of stay, number of admissions in the previous 12 months, urgent or emergent index hospitalization, total number of medications on discharge, opiate medication use at discharge, anticoagulation at discharge, Elixhauser comorbidity index, hemoglobin level at discharge, sodium level at discharge, hematologic neoplasm, metastatic neoplasm, ovarian neoplasm, congestive heart failure, and liver disease. In the multivariable analysis, the following risk factors remained significantly and independently associated with a potentially avoidable readmission: total number of medications at discharge, liver disease, sodium level at discharge, and hemoglobin level at discharge (Table 2).

The five most frequent primary diagnoses at readmission were neoplasm, infection, nutritional and metabolic disorder, GI disorder, and renal failure (Table 3). Of note, these were five of the top six primary diagnoses for the index hospitalization (Table 1). The median time of occurrence of 30-day potentially avoidable readmissions was 10 (IQR, 9 to 11 ) days versus 13 (IQR, 12 to 14 ) days for 30-day unavoidable readmissions $(P<.001)$. The median time of 30 -day potentially avoidable readmission varied from 4 to 12 days according to the primary diagnosis of readmission (Table 3). Readmissions due to adverse drug events, GI disorders, renal failure, or infection tended to

Table 2. Multivariable Analysis of Risk Factors for 30-Day Potentially Avoidable Readmission in Patients With Cancer

\begin{tabular}{|c|c|c|}
\hline Variable & Odds Ratio ( $95 \% \mathrm{Cl}$ ) & $P$ \\
\hline Urgent/emergent index hospitalization & 1.34 (0.82 to 2.19$)$ & .24 \\
\hline No. of admissions in the previous year* & 1.05 (0.99 to 1.12$)$ & .10 \\
\hline Total No. of medications at discharge* & $1.03(1.01$ to 1.06$)$ & $.02 \dagger$ \\
\hline Anticoagulation therapy at discharge & 1.36 (0.99 to 1.85$)$ & .05 \\
\hline Elixhauser comorbidity index, per 1-unit increase & 1.00 (0.99 to 1.01$)$ & .96 \\
\hline Congestive heart failure & 1.58 (0.96 to 2.59$)$ & .07 \\
\hline Ovarian neoplasm & $1.88(0.88$ to 4.00$)$ & .10 \\
\hline Hemoglobin, per $1 \mathrm{mmol} / \mathrm{L}$ decrease & $1.14(1.03$ to 1.25$)$ & $.01 \dagger$ \\
\hline Sodium level, per $1 \mathrm{mmol} / \mathrm{L}$ decrease & $1.04(1.00$ to 1.07$)$ & $.03+$ \\
\hline
\end{tabular}

*Per 1-unit increase.

†Statistically significant. 
Table 3. Median Time of Readmission According to the Cause of 30-Day Potentially Avoidable Readmissions ( $n=341)$

\begin{tabular}{|lcc|}
\hline Primary Diagnosis of Readmission & No. (\%) & $\begin{array}{c}\text { Median Time of } \\
\text { Readmission (IQR) }\end{array}$ \\
\hline Neoplasm & $67(19.7)$ & $10(4-19)$ \\
\hline Infection & $45(13.2)$ & $9(4-14)$ \\
\hline Nutritional or metabolic disorder & $17(5.0)$ & $11(6-14)$ \\
\hline Gl disorder & $16(4.7)$ & $6.5(4-14)$ \\
\hline Renal failure & $11(3.2)$ & $8(4-17)$ \\
\hline Arterial or venous thrombosis & $5(1.5)$ & $14(14-19)$ \\
\hline Adverse drug event & $5(1.5)$ & $6(6-17)$ \\
\hline Heart failure & $3(0.9)$ & $12(4-26)$ \\
\hline Other & $172(50.4)$ & $11(5-17)$ \\
\hline
\end{tabular}

Abbreviation: IQR, interquartile range.

occur earlier, whereas readmissions due to nutritional or metabolic disorders, heart failure, and thrombosis occurred later.

\section{DISCUSSION}

In this cohort of 2,916 patients with cancer, we found that almost $40 \%$ were readmitted within 30 days and that approximately one third of these readmissions were considered potentially preventable. Several factors were identified as independently associated with 30-day potentially avoidable readmission, including the total number of medications at discharge, liver disease, last sodium level, and last hemoglobin level before discharge. Of note, potentially avoidable readmissions occurred significantly earlier than unavoidable readmissions, with time to readmission varying substantially according to the readmission diagnosis.

Patients with cancer have a particularly high risk for readmission, ${ }^{2,13}$ but the characteristics of this population are not clear. The majority of the studies on readmission among patients with cancer looked at surgical patients with postoperative readmission for specific cancers. ${ }^{14-19}$ Much less is known about the readmission characteristics for general patients with cancer hospitalized within a medical or oncology service. $^{20-22}$

Many of the potentially avoidable readmissions in this study simply represent progression of disease or unavoidable adverse effects of treatment (and thus, are not truly preventable). However, as in other studies that used the SQLape algorithm, those with potentially avoidable readmissions should be thought of as a population enriched for patients whose readmissions might have been prevented with more-intensive transitional care activities.

The risk factors identified in this study seem plausible. The total number of medications likely represents a proxy for patient comorbidities and/or higher risk for readmission due to adverse drug events. Liver disease in patients with cancer may represent metastatic disease, which carries a poor prognosis as well as a risk for bleeding, infection, hepatic encephalopathy, and other complications of cirrhosis. Low sodium level is associated with poor outcomes in many disease states ${ }^{23-26}$ and may reflect a higher risk due to comorbidities that cause hyponatremia (eg, heart failure), chemotherapy-induced dehydration, or syndrome of inappropriate antidiuretic hormone secretion due to total cancer burden or lung or brain involvement. Finally, a low hemoglobin may reflect bone marrow suppression associated with certain chemotherapies, poor nutritional status, and/or anemia of chronic inflammation. Most of these risk factors are not modifiable, however, and we do not imply that by addressing these risk factors themselves, even if they are modifiable, postdischarge outcomes would be improved. However, these risk factors identify patients who may benefit from intensive transitional care interventions.

The study has several limitations. First, we included in the analysis only predictors easily obtainable from electronic data sources. We cannot exclude the presence of other important risk factors for readmission, such as the functional status of the patient. In addition, we were not able to capture the stage of the cancer. On the basis of the available data, we show that metastatic neoplasm was not an independent risk factor for 30day potentially avoidable readmission in multivariable analysis. This finding suggests that the stage may not be as important as expected, but we acknowledge that this coding is an imperfect proxy for cancer stage and that further studies are needed to explore this particular relationship. Second, no gold standard is available for the definition of preventable readmission. The identification of potentially avoidable readmission with the SQLape algorithm is not perfect, and whether the algorithm is any more or less diagnostic for oncology patients than any other category of hospitalized medical patients is unknown. However, SQLape has been used in numerous other studies; uses clear and logical criteria, which allow for reproducibility and reliability in the analysis of large databases; and is useful for identifying risk factors. The study 
may have been underpowered to identify all clinically important risk factors. We do not have information to draw conclusions about whether the risk factors for potentially avoidable readmission differ for patients readmitted to a different hospital from the site of their index hospitalization. Finally, this single-center study was from an academic hospital with a major cancer institute, and results might not be generalizable to other settings. Next steps could include detailed medical record review to determine the true preventability of these readmissions, identify potentially actionable risk factors unique to this population, and better understand the differences in time to readmission among various populations.

In conclusion, readmission in this large cohort of patients with cancer was frequent, with approximately one third of readmissions deemed potentially preventable. Risk factors associated with 30-day potentially avoidable readmission are the number of medications, liver disease, and low sodium and hemoglobin levels. Patients discharged with these factors could benefit from transitional care interventions. JOP

\begin{abstract}
Acknowledgment
J.D.D. was supported by the Swiss National Science Foundation and the Swiss Foundation for Medical-Biological Scholarships (grant PASMP3-142734). Presented in part at the 38th Annual Meeting of the Society of General Internal Medicine, Toronto, Ontario, Canada, April 22-25, 2015. We thank Yves Eggli for screening the database for potentially avoidable readmissions by using the SQLape algorithm. Dr Eggli is an associate physician at the Institute of Health Economics and Management of the University of Lausanne, Switzerland. He did not play a role in the design and conduct of this study, the analysis or interpretation of the data, or the preparation of this manuscript, and did not receive compensation for his help. None of the funding organizations were involved in the design and conduct of the study; collection, management, analysis, and interpretation of the data; preparation, review, or approval of the manuscript; and decision to submit the manuscript for publication.
\end{abstract}

\section{Authors' Disclosures of Potential Conflicts of Interest}

Disclosures provided by the authors are available with this article at ascopubs.org/journal/jop.

\author{
Author Contributions \\ Conception and design: Jacques D. Donzé, Jeffrey L. Schnipper \\ Financial support: Jacques D. Donzé \\ Administrative support: Jacques D. Donzé \\ Collection and assembly of data: Jacques D. Donzé \\ Data analysis and interpretation: Jacques D. Donzé, Stuart Lipsitz \\ Manuscript writing: All authors \\ Final approval of manuscript: All authors
}

Corresponding author: Jacques D. Donzé, MD, MSc, Department of Medicine, Bern University Hospital, Freiburgstrasse 4, Inselspital, 3001 Bern, Switzerland; e-mail: donze.author@gmail.com.

\section{References}

1. Donzé J, Lipsitz S, Bates DW, et al: Causes and patterns of readmissions in patients with common comorbidities: Retrospective cohort study. BMJ 347:f7171, 2013
2. Donzé J, Aujesky D, Williams D, et al: Potentially avoidable 30-day hospital readmissions in medical patients: Derivation and validation of a prediction model. JAMA Intern Med 173:632-638, 2013

3. Jones S, Wallis P: Effectiveness of a geriatrician in the emergency department in facilitating safe admission prevention of older patients. Clin Med (Lond) 13:561-564, 2013

4. Donzé J, Lipsitz S, Schnipper JL: Risk factors for potentially avoidable readmissions due to end-of-life care issues. J Hosp Med 9:310-314, 2014

5. Schnipper JL, Roumie CL, Cawthon C, et al: Rationale and design of the Pharmacist Intervention for Low Literacy in Cardiovascular Disease (PILL-CVD) study. Circ Cardiovasc Qual Outcomes 3:212-219, 2010

6. Kripalani S, Roumie CL, Dalal AK, et al: Effect of a pharmacist intervention on clinically important medication errors after hospital discharge: A randomized trial. Ann Intern Med 157:1-10, 2012

7. Halfon P, Eggli Y, van Melle G, et al: Measuring potentially avoidable hospital readmissions. J Clin Epidemiol 55:573-587, 2002

8. SQLape: Potentially avoidable readmissions, 2013. http://www.sqlape.com/ READMISSIONS.htm

9. Halfon P, Eggli Y, Prêtre-Rohrbach I, et al: Validation of the potentially avoidable hospital readmission rate as a routine indicator of the quality of hospital care. Med Care 44:972-981, 2006

10. Hasan O, Meltzer DO, Shaykevich SA, et al: Hospital readmission in general medicine patients: A prediction model. J Gen Intern Med 25:211-219, 2010

11. Boult C, Dowd B, McCaffrey D, et al: Screening elders for risk of hospital admission. J Am Geriatr Soc 41:811-817, 1993

12. Silverstein MD, Qin H, Mercer SQ, et al: Risk factors for 30-day hospital readmission in patients $\geq 65$ years of age. Proc Bayl Univ Med Cent 21:363-372, 2008

13. Shu CC, Lin YF, Hsu NC, et al: Risk factors for 30-day readmission in general medical patients admitted from the emergency department: A single centre study. Intern Med J 42:677-682, 2012

14. Ogawa F, Satoh $Y$, lyoda A, et al: Clinical impact of lung age on postoperative readmission in non-small cell lung cancer. J Surg Res 193:442-448, 2015

15. Sutton JM, Wima K, Wilson GC, et al: Factors associated with 30-day readmission after restorative proctocolectomy with IPAA: A national study. Dis Colon Rectum 57:1371-1378, 2014

16. Sadot E, Brennan MF, Lee SY, et al: Readmission after pancreatic resection: Causes and causality pattern. Ann Surg Oncol 21:4342-4350, 2014

17. Kimbrough CW, Agle SC, Scoggins CR, et al: Factors predictive of readmission after hepatic resection for hepatocellular carcinoma. Surgery 156:1039-1046, 2014 18. Mlodinow AS, Ver Halen JP, Lim S, et al: Predictors of readmission after breast reconstruction: A multi-institutional analysis of 5012 patients. Ann Plast Surg 71: 335-341, 2013 [Erratum: Ann Plast Surg 71:627, 2013]

19. Ahmad SA, Edwards MJ, Sutton JM, et al: Factors influencing readmission after pancreaticoduodenectomy: A multi-institutional study of 1302 patients. Ann Surg 256:529-537, 2012

20. Chiang LY, Liu J, Flood KL, et al: Geriatric assessment as predictors of hospital readmission in older adults with cancer. J Geriatr Oncol 6:254-261, 2015

21. Ji H, Abushomar $\mathrm{H}$, Chen $\mathrm{XK}$, et al: All-cause readmission to acute care for cancer patients. Healthc Q 15:14-16, 2012

22. Weaver C, Schiech L, Held-Warmkessel J, et al: Risk for unplanned hospital readmission of patients with cancer: Results of a retrospective medical record review. Oncol Nurs Forum 33:E44-E52, 2006

23. Zilberberg MD, Exuzides A, Spalding J, et al: Epidemiology, clinical and economic outcomes of admission hyponatremia among hospitalized patients. Curr Med Res Opin 24:1601-1608, 2008

24. Scherz N, Labarère J, Méan M, et al: Prognostic importance of hyponatremia in patients with acute pulmonary embolism. Am J Respir Crit Care Med 182: 1178-1183, 2010

25. Nootens M, Kaufmann E, Rector T, et al: Neurohormonal activation in patients with right ventricular failure from pulmonary hypertension: Relation to hemodynamic variables and endothelin levels. J Am Coll Cardiol 26:1581-1585, 1995

26. Sajadieh A, Binici Z, Mouridsen MR, et al: Mild hyponatremia carries a poor prognosis in community subjects. Am J Med 122:679-686, 2009 


\section{AUTHORS' DISCLOSURES OF POTENTIAL CONFLICTS OF INTEREST}

\section{Risk Factors and Patterns of Potentially Avoidable Readmission in Patients With Cancer}

The following represents disclosure information provided by authors of this manuscript. All relationships are considered compensated. Relationships are self-held unless noted. I = Immediate Family Member, Inst = My Institution. Relationships may not relate to the subject matter of this manuscript. For more information about ASCO's conflict of interest policy, please refer to www.asco.org/rwc or ascopubs.org/journal/jop/site/misc/ifc.xhtml.

\section{Jacques D. Donzé}

No relationship to disclose

\section{Stuart Lipsitz}

No relationship to disclose
Jeffrey L. Schnipper

Research Funding: Sanofi 


\section{Appendix}

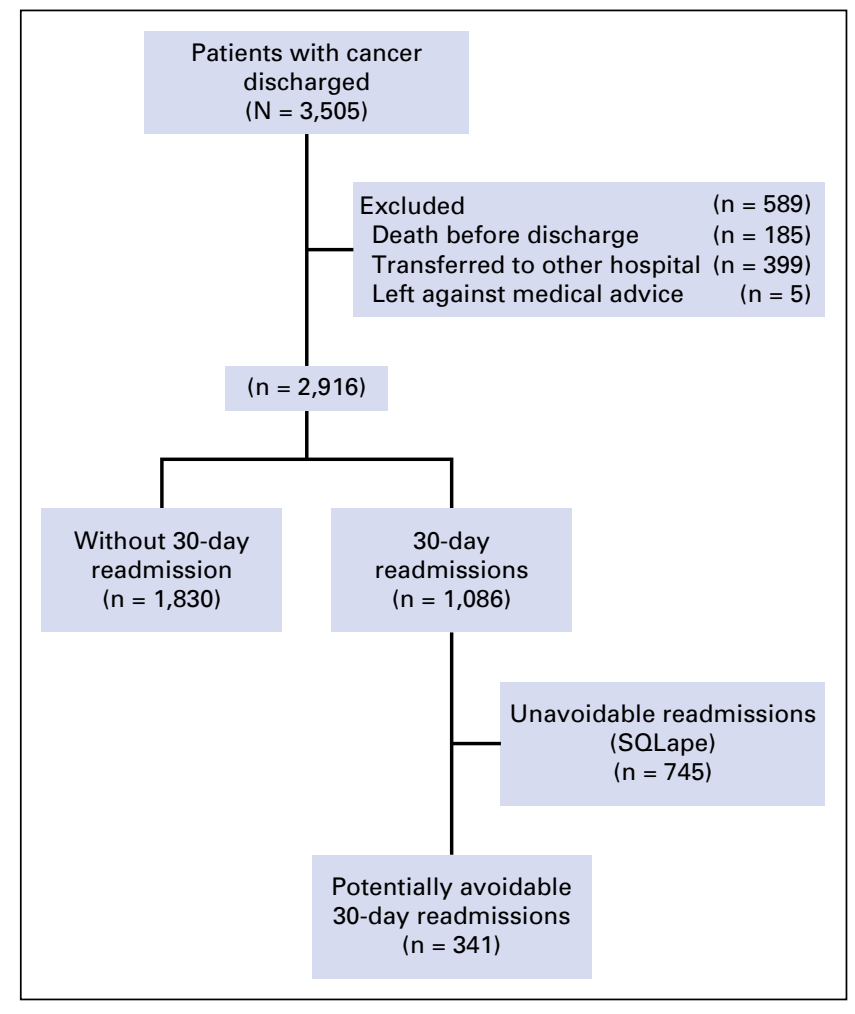

FIG A1. Study flow diagram describing the readmissions of all consecutive discharges from the oncology division of an academic tertiary medical center in Boston, MA, between July 1, 2009, and June 30, 2010. 